\title{
Sealing and dentin bond strength of adhesive systems in selected areas of perfused teeth ${ }^{\text {i⿱ }}$
}

\author{
N. Escribano, O. Del-Nero, J.C. de la Macorra* \\ Faculty of Dentistry, Department of Conservative Dentistry, Complutense University, Plaza de Ramón y Cajal s/n, 28040 Madrid, Spain
}

\begin{abstract}
Objectives: (1) Test the sealing capacity and tensile strength of various adhesive systems: Scotch Bond 1 (Single Bond in USA)(SB1) + Z100, Syntac Sprint (SYN) + Tetric Ceram, Prime and Bond 2.1 (PB21) + TPH Spectrum, F2000 (Adhesive + Compomer) (F2000) and Optibond Solo (OPT) + Prodigy, to perfused teeth $\left(30 \mathrm{~cm}\right.$ distilled $\left.\mathrm{H}_{2} \mathrm{O}\right)$, in select areas. (2) Determine the relationship between sealing ability and size of adhesive area (BA) with tensile strength (TS).

Methods: Segments of human third molars $(n=67)$ sealed with amalgam on their coronal side were connected to a perfusion system ( $30 \mathrm{~cm}$ distilled $\mathrm{H}_{2} \mathrm{O}$ ), to measure the basal permeability. Access cavities were drilled through the amalgam to expose dentin (area range: $8.7-20.8 \mathrm{~mm}^{2}$ ) and measure the permeability of that area. Cavities were sealed with one of the test materials and permeability measured prior to tensile testing. The percentage of decrease in permeability (PPD) that occurred with the different combinations of materials was also measured.

Results. Tensile strength: there were no statistically significant differences among groups. Permeability: ANOVA showed that there were statistically significant differences in the mean $\%$ decrease in permeability (PPD) among groups $(p=0.018)$. Student-Newman-Keuls test $(p<0.05)$ detected that the mean PPD of F2000 ranked statistically higher than that of SB1. Relationship among variables: a linear correlation was found defined by the formula TS $=7.47+0.008 \times \mathrm{PPD}-0.23 \times \mathrm{BA}$, with a significance of the model $p=0.0097$.

Significance: None of the materials studied reduced permeability up to $100 \%$. They all produced low tensile bond strengths (maximum mean value of groups: 5.51 MPa (OPT)). (C) 2001 Academy of Dental Materials. Published by Elsevier Science Ltd. All rights reserved.
\end{abstract}

Keywords: Perfusion; Dentin permeability; Sealing; Tensile bond strength

\section{Introduction}

One of the desirable conditions of restorative adhesive materials is that they should seal the interface in a similar manner to what occurs naturally. This is justified because such sealing would prevent the passage of harmful stimuli and substances from the oral environment to the tooth. Bacterial penetration could lead to pulpal irritation [1-3]. In addition, a non-physiological sealing would permit greater movement of the dentin fluid in the tubules that could result in dentin hypersensitivity if the hydrodynamic stimuli reach a sufficient threshold $[1,2,4,5]$.

Dentin wetness from the tubular fluid plays a major role in adhesion. Several in vitro studies [2-11] have shown that adhesion is reduced when bonding to humid substrates. To predict the effectiveness of the restorative material when replacing the lost dental structures, attempts have been made to find a correlation between the sealing capacity and adhesion strength of different adhesive restorative materials. Different authors [1,3,4,5,11-15] have disagreed on this point. For some researchers $[1,3,5,12,13,15]$ it is clear that dentin permeability (i.e. the sealing ability) is inversely related to the magnitude of bond strengths. For other authors [4,11] there is not such a clear relation, at least for specific materials that might benefit from a moist environment. For these authors $[4,11]$ the adhesion strengths would depend on the hybridization mechanism, regardless of whether or not the interface is sealed.

For the newer dentin adhesives that have a high hydrophilic capacity, the presence of moisture in the substrate is desirable. Being physiological dentin in a moist substrate it is reasonable to consider these hydrophilic adhesives less technique sensitive because they work well on naturally wet surfaces $[1,7,8,10,16]$. Dentin contains 30,000 tubules $/ \mathrm{mm}^{2}$, 


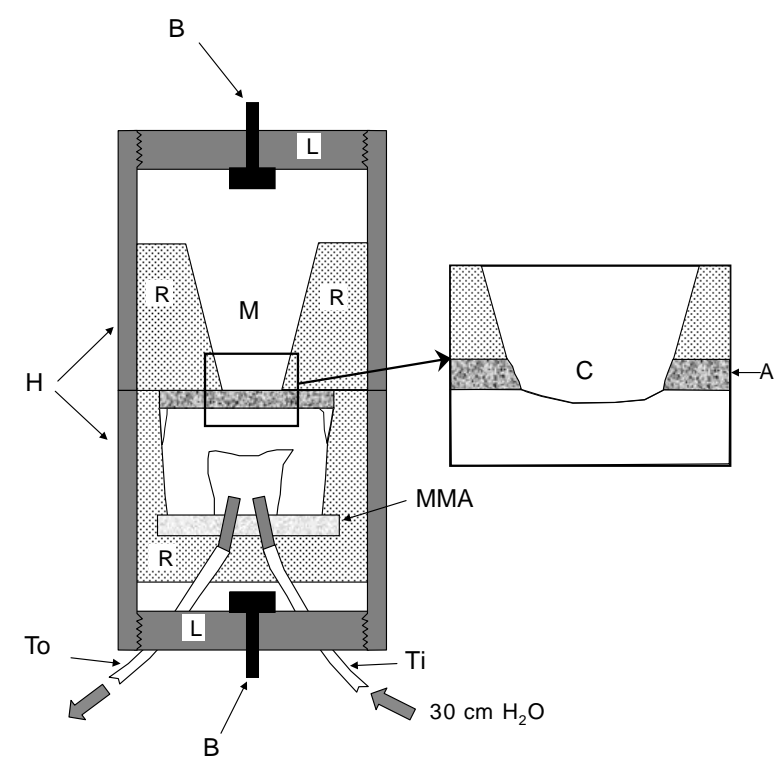

Fig. 1. R, embedding (lower) and matrix resins (upper); A, amalgam layer; C, cavity; M, matrix; B, bolts to traction machine; L, Lid; MMA, methylmethacrylate base; $\mathrm{Ti}$, input tube; To, output tube; $\mathrm{H}$, holder.

with a mean anatomic diameter of $1 \mu \mathrm{m}$. Both the diameter of the tubules and the number of tubules by unit area decrease as we approach the enamel dentin junction. The tubules do not represent open channels, but under scanning electronic microscopy they show an image of a fibrous matrix in the form of a hydrogel [14]. This matrix restricts the hydraulic conductance [14] of the extracellular fluid, which is rich in plasmatic proteins. The pressure of this fluid is in a range of 7-40 cm of water [8].

In previous studies, we attempted to find a relationship between the sealing capacity and bond strength of different dentin adhesive systems [17]. It was observed that the permeability varied according to the type of material used, and that this could not be entirely attributed to the water sorption of the material. None of the materials studied was able to reduce permeability completely. In addition, the dentin bond strengths obtained in that study were notably low. This was attributed to the large areas studied, since dentin bond strengths depend on the size of the adhesive area. According to Sano et al. [18] small areas are associated with high adhesion strengths, whilst wider areas show lower adhesive strengths. This phenomenon could be explained by Griffith's theory [18], which establishes that the probability of the existence of defects and/or fatigue will be greater in a material that has big dimensions rather than a smaller one. Failure in adhesion could then be attributed to the presence of such defects, that would act as initiators of the rupture, in specimens with large adhesive areas. In our study, these low adhesion values could not be related with the sealing capacity of the material.

The objectives of the present study are to assess the sealing capacity and tensile bond strengths with reference to the bonded area surface.

\section{Material and methods}

Recently extracted third molars $(n=67)$ without caries or restorations were used in this study. They were preserved at room temperature in a 70\% ethanol solution [19] until used. The teeth were cut at cervical level using a diamond disk to extract the contents of the pulpal chamber. This extraction was accomplished using dissection tweezers without touching the chamber walls. Enamel was removed to expose dentin using a new diamond bur and abundant water spray. Both the cut areas were lightly polished with 1200-grit sandpaper in a polishing machine (Struers Dap-7, Denmark). Then the tooth was glued at its cervical zone to a methacrylate base (MMA in Fig. 1) by a cyanoacrylate general-purpose adhesive (Super Glue 3, Loctite, Spain). The methacrylate base had two holes where two metal catheters were fitted (Ti and To in Fig. 1) entering the pulpal chamber. The passage of the catheters through the methacrylate base was sealed with acrylic resin (Duralay, Reliance, USA).

Lateral areas of the specimens were covered with a layer of nail polish to prevent leakage. The exposed dentin area was treated with $37 \%$ orthophosphoric acid (Total Etch, batch B28357, Ivoclar/Vivadent, Schaan, Liechtenstein), washed thoroughly and excess water removed with absorbent paper, as recommended by De Goes et al. [20]. The Scotchbond Multi-Purpose System (3M, Dental Products Division, batch no 70-2010-1232-8) was then applied to seal the entire exposed area with amalgam (Amalcap Plus Nongamma2, Ivoclar/Vivadent. batch 532288NN) (A in the enlarged detail of Fig. 1), forming a layer of around $1 \mathrm{~mm}$ thick. After $24 \mathrm{~h}$, this area was polished with 1200 sandpaper under abundant water refrigeration.

The assembly was included in a resin matrix (Cronolita 11700/3115, Plastiform, Spain) (R in Fig. 1) contained in a steel holder (H in Fig. 1). At the same time, a counterpart was prepared in another holder similar to the one above. This counterpart would act as matrix ( $\mathrm{M}$ in Fig. 1) via a central, conical perforation. Once the resin was polymerized, the exposed amalgam and the counterpart resin areas were again polished.

The specimen was connected to a simulated perfusion system made up of a column of $30 \mathrm{~cm}$ of distilled water, which reproduces the intrapulpal pressure. The column was connected to a plastic tube that transmitted the fluid to the pulpal chamber through Ti (Fig. 1). A non-graded micropipette $(10 \mu l)$ (Microcaps, Drummond Scientific Co., USA) was interposed. Using syringes in the micropipette, an air bubble was introduced. The movement of this bubble allowed the amount of flow to be measured. Via the other catheter (To in Fig. 1) the air was extracted from the chamber until it was saturated with liquid. Upon removing this air, this catheter was sealed.

The first measurement of permeability was made with the specimen covered with silver amalgam. The results obtained were taken as baseline, to be compared with the ones that 
Table 1

Materials used in the study

\begin{tabular}{|c|c|c|c|c|c|c|}
\hline Group & Adhesive & Batch & Composition & Restoration & Batch & Manufacturer \\
\hline & Scotch bond Multi-purpose & $70-2010-1232-8$ & \multicolumn{4}{|c|}{$\begin{array}{l}\text { Activator (component 1.5): ethanol-based solution of sulfinic acid salt, photoinitiator } \\
\text { Primer (component 2): aqueous solution of HEMA, polyalkenoic acid copolymer } \\
\text { Adhesive (component 3): bis-GMA, HEMA, photoinitiator } \\
\text { Catalyst (component 3.5): Bis-GMA, HEMA, peroxide chemical initiator. }\end{array}$} \\
\hline SB1 & Scotch Bond $1^{\mathrm{a}}$ & 199770515 & $\begin{array}{l}\text { Bis-GMA, HEMA, ethanol, } \\
\text { water, diacrylates, } \\
\text { photoinitiator, copolymer of } \\
\text { polyalkenoic acid }\end{array}$ & $\mathrm{Z} 100$ & 19970910 & 3M Dental Products Division \\
\hline SYN & Syntac Sprint & A06731 & $\begin{array}{l}\text { Poliacrylic modified } \\
\text { methacrylates, HEMA, } \\
\text { water, acetone maleic acid, } \\
\text { fluoride, catalysts }\end{array}$ & Tetric Ceram & A02383 & Ivoclar/Vivadent \\
\hline $\mathrm{PB} 21$ & Prime\&Bond 2.1 & 9802001066 & $\begin{array}{l}\text { PENTA, BIS-GMA urethane } \\
\text { modified, acetone, } \\
\text { camphorquinone, } \\
\text { elastomeric resins }\end{array}$ & TPH Spectrum & 9712000722 & Dentsply/DeTrey \\
\hline F2000 & F2000 Adhesive & 19970929 & $\begin{array}{l}\text { Component A: HEMA, } \\
\text { copolymer of polyalkenoic } \\
\text { acid, water, ethanol } \\
\text { photoinitiator } \\
\text { Component B: } 1-4 \% \text { maleic } \\
\text { acid, water }\end{array}$ & F2000 Compomer & 19970913 & $3 \mathrm{M}$ \\
\hline OPT & OptiBond SOLO & 706472 & $\begin{array}{l}\text { BIS-GMA, HEMA, GPDM, } \\
\text { ethanol, silica, barium } \\
\text { crystals, sodium fluoride }\end{array}$ & Prodigy & 801285 & Sybron/Kerr \\
\hline
\end{tabular}

${ }^{\text {a }}$ Marketed as single bond in USA.

were obtained at subsequent phases. A second permeability measurement was made after drilling a cavity (C in Fig. 1) through the amalgam with a doughnut diamond bur, so that the central part of the amalgam layer was removed until dentin was exposed. All cavities were drilled in a round configuration, with a diameter of approximately $4 \mathrm{~mm}$ and divergent walls, coinciding with the central perforation of the counterpart and conditioned (Total Etch, batch B28357, Ivoclar/Vivadent, Schaan Liechtenstein) to eliminate the smear produced by the bur and excess water removed as cited above. All cavities were u-shaped with a mean depth of $0.28 \mathrm{~mm}(\mathrm{SD}=0.08 \mathrm{~mm})$.

After the second permeability measurement, the dentin area was re-conditioned with $37 \%$ orthophosphoric acid (Total Etch, batch B28357, Ivoclar/Vivadent, Schaan Liech-

Table 2

Results ( $n$, number of cases; $m$, mean; SD, standard deviation; PPD, $\%$ decrease in permeability after sealing; BA, bonded area range; TS, tensile strength; superscripts A,B-PPD comparisons (SNK groups, $p<0.05$ ))

\begin{tabular}{|c|c|c|c|c|c|c|c|c|}
\hline \multirow[t]{2}{*}{ Material } & \multicolumn{3}{|c|}{ PPD (\%) } & \multicolumn{2}{|c|}{$\mathrm{BA}\left(\mathrm{mm}^{2}\right)$} & \multicolumn{3}{|c|}{ TS (MPa) } \\
\hline & $n$ & $m$ & SD & $\min$ & $\max$ & $n$ & $m$ & SD \\
\hline $\mathrm{SB} 1^{\mathrm{A}}$ & 11 & 19.35 & 34.32 & 10.0 & 19.5 & 9 & 3.38 & 1.09 \\
\hline $\mathrm{SYN}^{\mathrm{A}, \mathrm{B}}$ & 12 & 30.35 & 26.12 & 11.5 & 20.8 & 12 & 3.93 & 1.97 \\
\hline $\mathrm{PB} 21^{\mathrm{A}, \mathrm{B}}$ & 20 & 41.61 & 20.95 & 9.5 & 13.7 & 11 & 5.35 & 2.56 \\
\hline $\mathrm{OPT}^{\mathrm{A}, \mathrm{B}}$ & 10 & 40.38 & 27.74 & 9.3 & 15.7 & 9 & 5.51 & 1.52 \\
\hline $\mathrm{F} 2000^{\mathrm{B}}$ & 14 & 54.52 & 23.32 & 8.7 & 13.4 & 5 & 5.48 & 1.07 \\
\hline
\end{tabular}

tenstein) to eliminate any possible deposit or debris due to filtration. Excess water was again removed as cited before. Different bonding agents were applied in that zone with their corresponding restorative materials, through the counterpart that adjusts perfectly to the test piece containing the specimen. The third measurement of permeability was then carried out. Percentage permeability decrease (PPD) was calculated with the formula

$\mathrm{PPD}=\frac{100[\mathrm{CP}-\mathrm{RP}]}{\mathrm{CP}}$,

where PPD is the percentage of permeability decrease, CP the permeability of the drilled cavity in $\mu \mathrm{l} / \mathrm{min}$, and RP is the permeability of the restored cavity in $\mu \mathrm{l} / \mathrm{min}$.

All materials (Table 1) were applied following the manufacturer's instructions. After concluding the permeability measurements, the assembly formed by the test piece and the counterpart was removed from the perfusion system, bolts were coupled (B in Fig. 1) to the lids of the metal holders (L in Fig. 1) to permit traction and taken to a universal mechanical testing machine (Hounsfield HTI, Great Britain), with a $500 \mathrm{~N}$ load cell, working at a speed of $1 \mathrm{~mm} /$ min, to determine the tensile strengths (TS). After the specimen fractured it was inspected under a stereoscopic microscope with an image analyzer (Leica Qwin Q500 IW) to determine the area of adhesion and the presence of adhesive or cohesive fracture.

ANOVA and Student-Newman-Keuls $(p<0.05)$ tests 
Table 3

Significance of coefficients

\begin{tabular}{lcl}
\hline & Coefficient & Significance \\
\hline Constant & 7.47 & 0.00000018 \\
PPD & 0.008 & 0.33 \\
BA & -0.227 & 0.0095 \\
\hline
\end{tabular}

were carried out to find, if any, statistically significant differences in the results of the percentage decrease in permeability after restoration (PPD) for each material and in the TS results.

A linear formula was tested (SPSS ver 9.0.1, Chicago, USA) in the form $\mathrm{TS}=a+b \times \mathrm{PPD}+c \times \mathrm{BA}$, looking for the best $a, b$ and $c$ coefficients.

\section{Results}

Table 2 gives details of the PPD, bonded area range and TS values, per material. The TS data were discarded in some specimens for reasons that altered the development of the test (i.e. specimen dislodging, cohesive tooth or material fractures).

\subsection{Permeability}

ANOVA test showed there were statistically significant differences in PPD in the materials studied $(p=0.018)$. Student-Newman-Keuls test $(p<0.05)$ identified two homogeneous groups: A (SB1, SYN, PB21\&OPT) and B (SYN, PB21, OPT\&F2000).

\subsection{Traction}

The TS results ranged between 0.97 and $10.13 \mathrm{MPa}$, corresponding to specimens of groups SYN and PB21, respectively.

ANOVA test showed there were no statistically significant differences in TS means in the groups studied.

\subsection{Relationship among variables}

A linear relationship was calculated with TS as the dependent variable and PPD and BA as independent ones. The resultant formula was $\mathrm{TS}=7.47+0.008 \times \mathrm{PPD}-0.227 \times$ BA. The ANOVA test showed that significance of the model was $p=0.0097(r=0.411)$. Table 3 shows significance values of coefficients in the above formula.

\section{Discussion}

Newer adhesive and restorative materials have been developed, among other reasons, to reduce the possibility of marginal leakage that could lead to pulpal damage due to external agents. For this reason, these materials have been designed to produce adhesion to dentin under critical moisture conditions [13].

Generally, colorimetric methods have been used to show the sealing capacity of different restorative materials. Measurements are usually made determining the depth of penetration of the dye in one or more sections of a restored tooth [21]. One of the problems with this method is that it renders qualitative rather than quantitative data $[12,15,17]$. It shows the presence, or absence, of microleakage but not the amount. In addition, the data obtained from this method are difficult to compare and vary widely according to each specimen [15,21]. Another drawback in these methods is the high molecular weight of some dyes used as compared with that of the dentin fluid. The high molecular weight may actually impede the passage through the tubules and/or the interfaces. In other words, the absence of staining does not necessarily mean that there is no microleakage [17].

We, therefore, believe that this methodology is not valid to quantify the permeability of the restored teeth. Consequently, we have adopted a quantitative method to assess microleakage, based on various studies by Pashley et al. $[1,12,16,22]$ and by other authors [5,7,8]. The results obtained can be compared with the results of other studies. It is also possible to measure the permeability of an area of known dimensions [15].

System pressure in this study was $30 \mathrm{~cm}$ of distilled water. This water pressure was intended to mimic a physiologic pressure (actually unknown) that oscillates between 15 and $40 \mathrm{~cm}$ of water, depending on authors, methodology and animal species [23-27]. Nevertheless, some authors [28] consider that $32 \mathrm{~cm}$ would correspond with the pressure under pulpal inflammation.

Frequently, to force flow through dentin and then measure more easily the hydraulic conductance, higher pressures are applied [19,29-31] ranging from 200 to $700 \mathrm{~cm}$ water. These pressures are considered non-physiologic. Camps et al. [32] found that dentin permeability of human third molars varied depending on the pressure used. In this way, with low-pressure ranges (approx. $13 \mathrm{~cm} / \mathrm{w}$, considered into the physiologic range) permeability values were constant in time. With higher pressure (132, 271 or $408 \mathrm{~cm})$ a progressive decrease of hydraulic conductance was observed, probably due to displacement and impaction of tubular contents.

Previous studies $[1,3,13,15,33]$ are in agreement with our results, i.e. no material is able to produce a $100 \%$ decrease in dentin permeability. Supposedly, if a material seals the interface perfectly, the passage of fluid should drop to zero [5], but this occurred in none of our experiments. Our results show a wide range of permeability decreasing rates. This high variability may be due to the material studied and to the specimen used. This data coincides with the data obtained in similar work by our group [17], where it was also shown that permeability, though significantly reduced, persists.

All the materials studied, except F2000, require the use of acid etching to condition the dentin. All materials showed a 
decrease in post-sealing permeability that was comparable to that obtained in earlier studies with similar materials and similar specimen preparations $[1,12,13,33]$ although to a lesser extent than the very low $(80 \%)$ decrease rate obtained by Terkla et al. [33].

In the case of the F2000, acid etching was not used to condition the dentin before restoring, because the composition of the adhesive includes 5\% maleic acid. F2000 resulted in a decrease in permeability that was higher than that of the other materials studied (SB1). An explanation for this difference could be that F2000 restorations are placed after one acidic application (before first permeability measurement) and all other materials after two. This twofold etching may be responsible for an excessively thick demineralized dentin layer that is not completely impregnated by the adhesive. It would be necessary to analyze under high-resolution microscopy, the type of surface left after treatment with F2000 Adhesive/Primer and the type of interface it creates.

In our experimental design, residual permeability may be due to specimens leaking through the lateral, non-sealed areas, in spite of the layer of varnish that we used to make them waterproof. Another reason that could explain the presence of this residual permeability is the fact that the material tested is able to absorb water; but the amount of water absorbed by this means is imperceptible by our measuring system.

The first data on filtration obtained in this study (with the layer of amalgam intact) are taken as basal, so the variations in permeability recorded later must be exclusively due to the existence of cavities (of known dimensions) and the material tested.

Because of our experimental design, we have not started from the basal data of an intact dental surface, but one that is conditioned and sealed with amalgam, which could be the reason for the difference in results from other studies. The smeared layer produced when drilling the cavity through the amalgam must also be considered, although this is later eliminated with acid etching; maybe an excessive accumulation of smear layer partly blocks the tubules, which would explain why in some cases there is a reduction in permeability after drilling the cavity.

Regarding the possibility of permanence of traces of the adhesive layer of amalgam that could interfere with the bonding of tested materials, Prati et al. [34] found that Scotch Bond Multipurpose Plus (with primer and adhesive being the same as that in Scotch Bond Multipurpose System) hybrid layer thickness varied between 2 and $7 \mu \mathrm{m}$ dependent upon whether measurements were undertaken in superficial or deep intertubular dentin. Nakajima et al. [35] found, using the same product, that in humid conditions the sound dentin hybrid layer thickness was $3 \mu \mathrm{m}$ deep, while Marshall et al. [36] found this thickness to be of $4.5 \mu \mathrm{m}$ in superficial dentin and $7.8 \mu \mathrm{m}$ in deep dentin. Then, hybrid layer thickness will vary between 2 and $7.8 \mu \mathrm{m}$. When drilling the cavity we reach a mean depth of $280 \mu \mathrm{m}$ beyond the initial surface, thus ensuring the complete removal of adhered amalgam adhesive layer, although there is still the possibility of some tag of adhesive remaining.

The intention of the study was to compare different adhesive/composite combinations. This is the reason why we used different composite restorative resins in each group. It is known that a stiffer restorative material would render higher tensile bond strength results, because stress distribution would be on a larger area (at the limit, to the whole adhered surface) thus avoiding peeling stress [37]. However, our idea was precisely, to compare the behavior of whole systems, believing that the combination would be used more frequently than mixed materials.

With regard to the low adhesion strengths found, these also coincide with earlier experiments and they can be attributed mainly to the accumulation of defects or fatigue in large adhesive areas, according to Griffith's theory [18]. Furthermore, low TS found could be because dentin is maintained in moist conditions; at least this is what it seems when reviewing other studies with perfused specimens that also obtained low tensile strength values $[1,2,4-6,8,11,13,16,17,22]$. In these studies, the greater the initial permeability, the lower the final adhesion. In addition, an earlier study [13] reveals that the weak point in the adhesive system generally is located at the hybrid layer. The quality and morphology of that layer varies according to the dentin adhesive, which explains the different tensile values, without being influenced by the presence or absence of humidity.

With our methodology it is impossible to know where the planes of fracture of the detached samples were located. Debonding was qualified as adhesive-or, more precisely: not cohesive - when no parts of restorative material could be seen attached to the interface, nor the dentin bonding area obviously fractured. It would be helpful to know if such planes were within the hybrid layer or not, but this was beyond the scope of this work.

Our data render a predictive formula for tensile bond strength in the form $\mathrm{TS}=7.47+0.008 \times \mathrm{PPD}-0.227 \times \mathrm{BA}$. Its correlation coefficient $(r)$ is relatively low $(0.411)$ but the predictive power of the model is good enough $(p=$ $0.0097)$ to confirm that TS is governed by the sealing ability (PPD) and bonded area surface (BA).

Looking closely at the significance of coefficients (Table $3)$, PPD is affected by a positive coefficient (0.008) with low significance $(p=0.33)$, meaning that its contribution to explain the variance of TS is low. More valuable information is that BA is affected by a negative coefficient $(-0.227)$ with good significance $(p=0.0097)$.

Multiple regression analysis helps to determine what would be the effect of one of the independent variables on the dependent one, supposing the other one(s) are fixed. The formula should then be taken as a prediction of lower TS at a given PPD if larger areas are used. This is a statistical confirmation of known facts [18]. 
It has to be kept in mind that the range of areas is 8.7$20.8 \mathrm{~mm}^{2}$ (Table 2). This range can still be located in the asymptotic part of the previously published predictive formula [18] TS $=58.8-27.9 \log _{10}(\mathrm{BA})$, where sensible decreases of bonded areas produce little increases of tensile strength. For our range of areas, this formula predicts a range of TS of 32.6-22 MPa. Any discrepancy can be explained by differences in materials and methodology (our specimens were submitted to perfusion, while in the work by Sano et al. [18] they were not).

Our measuring system is designed to accept perfused specimens and to apply tensile loads to the adhered surfaces via the steel holders and the resin matrices. These structures are carefully aligned, but the resilience of the whole system can make torque forces appear at the adhesive surfaces. These torque forces would then cause the plane of failure to move to the next weakest zone. In this respect, a total of five samples were discarded because cohesive fractures were detected in the restorative material, at an apparent mean TS(SD) of 5.71(2.94) $\mathrm{MPa}$, which is below the predictable cohesive strength of the restorative materials used in our study. Four samples were discarded because of spontaneous dislodgment. Other discarded samples were because of failure of retention of the specimen in the resin holder or matrix.

Another possibility is that torque forces at the interface would transform tensile loads into peel ones. This would probably cause lower apparent tensile bond strength results.

\section{Conclusions}

- None of the materials studied produced a complete cessation of filtration. All permitted micro-leakage.

- Bonded area surface appears to be the most important predictive variable of tensile bond strength.

\section{Acknowledgements}

This study was funded by grants BE35/95 (Complutense University) to Dr Del-Nero, BE314/96 (Complutense University) to Dr Escribano and PM96-0044/96 (DGICYT, SPAIN) to Dr De la Macorra. The authors want to acknowledge Dr J. Perdigao for assistance in editing the English version and the manufacturers for generously providing all the materials tested.

\section{References}

[1] Pashley D, Derkson G, Tao L, Derkson M, Kalathoor S. The effects of a multi-step dentin bonding system on dentin permeability. Dental Materials 1988;4:60-3.

[2] Tao L, Pashley D. The relationship between dentin bond strengths and dentin permeability. Dental Materials 1989;5:133-9.

[3] Hansen S, Swift E, Krell K. Effect on permeability of dentin of two systems of dentin bonding. Journal of Esthetic Dentistry 1993;3:21-4 (Spanish ed.).

[4] Tagami J, Tao L, Pashley D. Correlation among dentin depth, permeability, and bond strength of adhesive resins. Dental Materials 1990;6:45-50.

[5] Prati C, Simpson M, Mitchem J, Tao L, Pashley D. Relationship between bond strength and microleakage measured in the same Class I restorations. Dental Materials 1992;8:37-41.

[6] Mitchem J, Terkla L, Gronas D. Bonding of resin dentin adhesives under simulated physiological conditions. Dental Materials 1998;4:351-3.

[7] Tao L, Pashley D. Dentin perfusion effects on the shear bond strengths of bonding agents to dentin. Dental Materials 1989;5:181-4.

[8] Prati C, Pashley D, Montanari G. Hydrostatic intrapulpal pressure and bond strength of bonding systems. Dental Materials 1991;7:54-8.

[9] Van Meerbeek B, Lambrechts P, Inokoshi S, Braem M, Vanherle G. Factors affecting adhesion to mineralised tissues. Operative Dentistry 1992;5((Spl. issue)):111-24.

[10] Kanka J. Adhesion of resins to moist substrates (I). Adhesion to dentin. Quintessence 1993;6:294-7 (Spanish ed.).

[11] Davidson C, Abdalla A, de Gee A. An investigation into the quality of dentine bonding systems for accomplishing a durable bond. Journal of Oral Rehabilitation 1993;20:291-300.

[12] Pashley D, O’Meara J, Williams E, Kepler E. Dentin permeability: effects of cavity varnishes and bases. Journal of Prosthetic Dentistry 1985;53:511-6.

[13] Prati C, Ferrieri P, Galloni C, Mongiorgi R, Davidson C. Dentine permeability and bond quality as affected by new bonding systems. Journal of Dentistry 1995;23:217-26.

[14] Linden L, Källskog Ö, Wolgast M. Human dentine as an hydrogel. Archives of Oral Biology 1995;40:991-1004.

[15] Pagliarini A, Rubini R, Rea M, Campese C, Grandini R. Effectiveness of the current enamel-dentinal adhesives: a new methodology for its evaluation. Quintessence International 1996;27:265-70.

[16] Pashley E, Tao L, Matthews W, Pashley D. Bond strengths to superficial, intermediate and deep dentin in vivo with four dentin bonding systems. Dental Materials 1993;9:19-22.

[17] Del-Nero O, de la Macorra JC. The sealing and dentin bond strength of adhesive systems. Operative Dentistry 1999;24:194-202.

[18] Sano H, Shono T, Sonoda H, Takatsu T, Ciucchi B, Carvalho R, Pashley D. Relationship between surface area for adhesion and tensile bond strength - evaluation of a micro-tensile bond test. Dental Materials 1994;10:236-40.

[19] Goodis HE, Marshall GW, White JM. The effects of storage after extraction of the teeth on human dentine permeability in vitro. Archives of Oral Biology 1991;36(8):561-6.

[20] De Goes MF, Ferrari Pachane GC, García-Godoy F. Resin bond strength with different methods to remove excess water from the dentin. American Journal of Dentistry 1997;10:298-301.

[21] Déjou J, Sindres V, Camps J. Influence of criteria on the results of in vitro evaluation of microleakage. Dental Materials 1996;12:342-9.

[22] Pashley E, Tao L, Derkson G, Pashley D. Dentin permeability and bond strengths after various surface treatments. Dental Materials 1989;5:375-8.

[23] Maita E, Simpson MD, Tao L, Pashley DH. Fluid and protein flux across the pulpodentine complex of the dog in vivo. Archives of Oral Biology 1991;36(2):103-10.

[24] Pashley DH, Nelson R, Pashley EL. In vivo fluid movement across dentine in the dog. Archives of Oral Biology 1981;26:707-10.

[25] Vongsavan N, Matthews B. Fluid flow through cat dentine in vivo. Archives of Oral Biology 1992;37(3):175-85.

[26] Ciucchi B, Bouillaguet S, Holz J, Pashley D. Dentinal fluid dynamics in human teeth, in vivo. Journal of Endodontics 1995;21(4):191-4.

[27] Van Hassel HJ. Physiology of the human dental pulp. Oral Surgery 1971;32(1):126-34.

[28] Gerzina TM, Hume WR. Effect of hydrostatic pressure on the 
diffusion of monomers through dentin in vitro. Journal of Dental Research 1995;74(1):369-73.

[29] Maroli S, Khera SC, Krell KV. Regional variation in permeability of young dentin. Operative Dentistry 1992;17:93-100.

[30] Pashley DH, Thompson SM, Stewart FP. Dentin permeability: effects of temperature on hydraulic conductance. Journal of Dental Research 1983;62(9):956-9.

[31] Pashley EL, Horner JA, Liu M, Kim S, Pashley DH. Effects of $\mathrm{CO}_{2}$ laser energy on dentin permeability. Journal of Endodontics 1992;18(6):257-62.

[32] Camps J, Giustiniani S, Déjou J, Franquin JC. Low versus high pressure for in vitro determination of hydraulic conductance of human dentine. Archives of Oral Biology 1997;42(4):293-8.

[33] Terkla L, Brown A, Hainisch A, Mitchem J. Testing sealing proper- ties of restorative materials against moist dentin. Journal of Dental Research 1987;66:1758-64.

[34] Prati C, Chersoni S, Mongiorgi R, Pashley DH. Resin-infiltrated dentin layer formation of new bonding systems. Operative Dentistry 1998;23:185-94.

[35] Nakajima M, Sano H, Zheng L, Tagami J, Pashley DH. Effect of moist vs. dry bonding to normal vs. caries-affected dentin with Scotchbond multi-purpose plus. Journal of Dental Research 1999;78(7):1298-303.

[36] Marshall SJ, Balooch M, Breunig T, Kinney JH, Tomsia AP, Inai N, Watanabe LG, Wu-Magidi IC, Marshall GW. Human dentin and the dentin-resin adhesive interface. Acta Materialia 1998;46(7):2529-39.

[37] Unterbrink GL, Liebenberg WH. Flowable resin composites as filled adhesives: literature review and clinical recommendations. Quintessence International 1999;30:249-57. 